\title{
Impact of Pedestrian Traffic on Saturation Rate of Protected Left-Turn at Urban Intersections
}

\author{
Yixin Chen*, Yulong He, Xiaoduan Sun \\ College of Metropolitan Transportation, Beijing University of Technology, Beijing, China \\ Email: ${ }^{*}$ chenyixin13@163.com, ylhe@bjut.edu.cn, xsun@louisiana.edu
}

Received 23 December 2014; accepted 16 January 2015; published 29 January 2015

Copyright (C) 2015 by authors and Scientific Research Publishing Inc.

This work is licensed under the Creative Commons Attribution International License (CC BY). http://creativecommons.org/licenses/by/4.0/

c) (i) Open Access

\section{Abstract}

Heavily congested intersections in metropolitan areas in China are facing unique problems due to high travel demand and a high degree of traffic law violations. Based on a study conducted by the authors of this paper, $93 \%$ of left-turn vehicles turning left in these areas were slowed in order to avoid conflict with pedestrians. Intertwined pedestrian and vehicular flows can significantly reduce the capacity of exclusive left-turn lane group through reducing saturation flow rate, which increases the congestion at intersections. This paper investigates how the saturation flow rate of exclusive left-turn lane group is affected by the characteristics of pedestrian flow. By analyzing the imagery data collected by video cameras installed at intersections, the research team is able to obtain the characteristics of both vehicular and pedestrian flows, such as speed and spatial locations. The average operating speed at the saturation flow rate with and without pedestrian traffic is used as a direct measurement to evaluate the effect of pedestrians. Based on the statistical analysis, the paper concludes that saturation flow rate is mainly affected by the position of pedestrian in the crosswalk (inside or outside of left-turn vehicle's trajectory), and the distance between the vehicle and pedestrians. In general, when the distance is less than four meters, the smaller the distance between vehicle and pedestrians, the larger the impact. However, there is no significant impact when the distance is larger than four meters. To accurately quantify the effect, the degree of pedestrian-vehicle impact is defined in four levels. The results show that the difference in the saturation flow rate between the best and the worst level could be $15.7 \%$, which clearly indicates how important it is to enforce pedestrian crossing behavior.

\section{Keywords}

Impact of Pedestrians, Exclusive Left-Turn Lane, Speed of Saturation Flow, Saturation Flow Rate

\footnotetext{
${ }^{*}$ Corresponding author.
}

How to cite this paper: Chen, Y.X., He, Y.L. and Sun, X.D. (2015) Impact of Pedestrian Traffic on Saturation Rate of Protected Left-Turn at Urban Intersections. Open Journal of Applied Sciences, 5, 22-31. 


\section{Introduction}

Signalized intersections in urban areas are particularly problematic because of not only high travel demands but also a high amount of pedestrians disregarding traffic signals. Based on our observations made at two typ- ical intersections in Beijing, about $63 \%$ of pedestrians at signalized intersections walk across the street while right-of-way is assigned by traffic signal to left-turning vehicles exclusively, and about $93 \%$ of left-turn vehicles are forced to slow in order to avoid pedestrians, undoubtedly affecting capacity of the lane group and consequently aggravating traffic congestion problems. Figure 1 shows a typical situation at a protected left-turn signal phase where vehicles are intertwined with pedestrians' crossing at the intersection. It is widely observed that more than $50 \%$ of pedestrians start walking across the street as soon as the signal turns green exclusively for left-turn vehicles. To avoid conflict with pedestrians, left-turn vehicles have to slow down or even totally stop to prevent an imminent crash. The speed slower than normal speed of left-turning vehicles could result in lower saturation flow rate. It is a serious problem for already heavily congested intersections in China.

Impatience, unawareness of the consequence of the action, lack of understanding traffic signal operation and/ or insufficient signal timing for pedestrian crossing may be the causes to this common illegal street crossing behavior in China. While poor pedestrian street crossing behaviors are largely ignored and uncontrolled in China, this problem has been recognized but few studies have been conducted on the impact of uncontrolled pedestrian to the saturation flow. Thus, the objective of this study is to investigate how the saturation flow is affected by illegal pedestrian crossing. It has been hoped that such studies can provide the evidence for decision makers on how to best manage congested signalized intersections in metropolitan areas.

\section{Literature Review}

Capacities of signalized intersections have been a topic of researches for many years. However, the research on the pedestrian effect has been limited. Generally speaking, pedestrian effects are studied in two aspects: 1) traffic flow is interrupted by pedestrians, and 2) the speed and saturation flow rate decreases due to pedestrians' interference.

Some researches focused on the interruption to the traffic flow by pedestrians. The study by Viney and Pretty [1] described three possible pedestrian cases: 1) vehicle traffic was cut off with zero flow rate; 2) vehicles passed through pedestrians when there was an acceptable headway gap; 3) vehicles flow at saturation rate was not affected by stopped pedestrian. Juan Enrique and Juan Carlos [2] established a regression model to study the pedestrian influence on saturation flow for turning lanes (left lane and right lane). Based on their study, the influential factors contained intersection turning radius, lane type and width, proportion of turning vehicles, existence of public transit stops, and the pedestrian volume. Their study results showed that interaction between pedestrians and turning vehicles had a large impact on vehicle delay. In 2008, Chen developed a model to investigate the capacity at intersection conflict zones based on the pedestrian behavior analysis and characteristics of pedestrian-vehicle conflicts [3]. All the studies in this area focused on time headway of vehicle flow and how pedestrians utilize the time headway to cross a street.

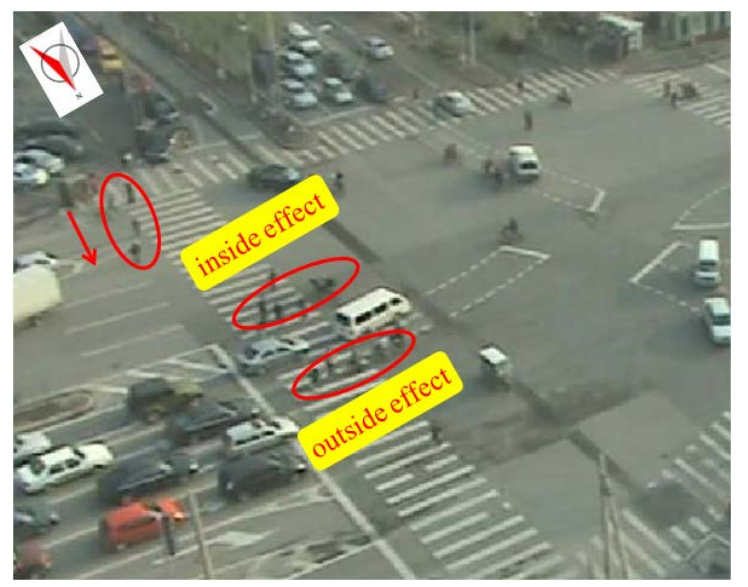

Figure 1. Pedestrian movement during protected left-turn phase. 
There are not many researches on the reduced speed and the reduced left-turn saturation flow rate caused by pedestrian crossings. A reduction factor (RF) has been commonly used to reflect the effect of pedestrians on vehicular flow. The Fundamentals of Traffic Engineering [4] gave the RF a constant value 0.9. Wei et al. [5] [6] calculated the value of reduction factors based on the initial reaction time of drivers, starting delays or lost time of the first few vehicles and the minimum headway. Highway Capacity Manual 2010 edition [7] calculated the occupied time of pedestrians to the conflict points based on pedestrian flow rate, and provides a formula for RF estimation by traffic volume.

Using the gap acceptance statistical analysis, Xu and Wang developed a model of influence on the straight vehicles. The result showed that speed of vehicles was affected poorly by pedestrians [8]. Based on vehicle intersection travel time and pedestrian volume, Chen classified signalized intersections serving both vehicular and pedestrian flows into 3 groups (lowest, moderate, and highest) to reflect individual intersection performance [9]. A study on the effect of pedestrian flow on vehicular operation at signalized intersections showed that vehicle speeds subject to a normal distribution when they were not affected by pedestrian flow and vehicular speed decreases when the vehicles' maneuvers were affected by pedestrian traffic [10]. By considering the distance between pedestrians and vehicles as an influential factor, Andras investigated vehicle speeds under free flow conditions while approaching a non-signalized mid-block crossing on a two-lane arterial road [11]. His study results showed that three out of four drivers maintain the same speed or accelerate when approaching the crossing and only one out of four vehicles slowed down or braked at a distance 50 to 40 meters before the crossing. The vehicle speed was affected by the distance between pedestrians and vehicles, the traffic volume and etc. The number of pedestrians was not considered in that paper.

In summary, many previous researches have revealed that vehicular flow, specifically the operating speed, is significantly affected by pedestrian traffic. The level of impact mainly depends on pedestrian volume and distance between pedestrians and vehicles. In China, the vehicle flow under a protected left-turn phase is commonly affected by pedestrians crossing at signalized intersections, which is a unique problem in many emerging or developing countries. Therefore, this paper aims to investigate how the saturation flow rate for protected leftturn phase is affected by the illegal but largely unenforced pedestrian crossings.

\section{Data Collection}

To investigate the problem, the imagery data has been collected by a camera placed at a corner of the intersection in order to get an accurate bird's-eye-view. This typical urban four-way intersection operates with a fourphase signal plan with a protected left-turn phase for both heavily traveled streets. Due to high traffic demand, the single, three meters wide and almost 100 meters long exclusive left turn lane on both streets is always fully occupied at peak time during other signal phases. The traffic volume passing through this intersection in 12 hours (7:00 - 19:00) is about 50,000 vehicles. During the rush hours the majority of left turning vehicles have to wait for more than one signal cycle to pass the intersection, which is much longer than normally observed during rush hours in other cities or countries.

The data collection lasted for three consecutive workdays. The targeted cross walk is 40 meters long and five meters wide. The effect of pedestrian movement was investigated on the right and left sides of vehicles separately. The right side is defined as outside and left side as inside. The relative positions of pedestrians and vehicles at the intersection were calculated by a positioning software every half second. The intersection and coordinatesystem are illustrated in Figure 2.

With the collected imagery data and the spatial data from processing software, the following variables are obtained: speed of every left-turn vehicle at the protected signal phase, positions of each moving vehicle, the distance between a vehicle and closest pedestrians on both sides and pedestrian volume. The data were collected under good weather with only small vehicles (excluding the data from the protected phase when buses, large trucks and U-turn vehicles are present) in order to derive the saturation flow rate under the prevailing condition. This was the same saturation rate estimation method introduced by the Highway Capacity Manual (HCM): the vehicle data collection start at the fourth car, which is:

$$
h_{t}=\frac{t_{n}-t_{4}}{n-4}, v_{f}=\frac{1}{n} \sum_{4}^{n} v_{i}
$$

where: 


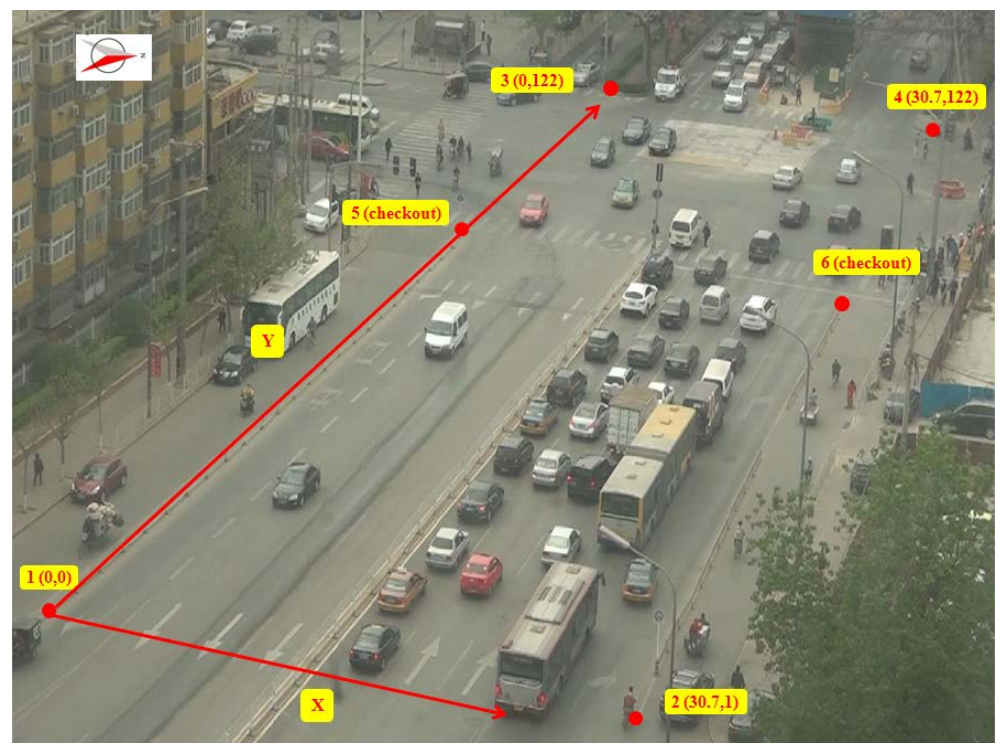

Figure 2. Layout of the investigated intersection.

$h_{t}$ : time-headway of the saturation flow;

$t_{n}$ : the time of the last car in the queue when it passes through the stop line;

$t_{4}$ : the time of the fourth car in the queue when it passes through the stop line;

$n$ : number of cars.

The saturation flow speed is calculated as the average speed from the fourth car to the last car in queue passing though the stop line.

The formula to calculate the saturation flow speed is:

$$
v_{f}=\frac{1}{n} \sum_{4}^{n} v_{i}
$$

where:

$v_{f}$ : the speed of left-turn saturate flow, the average speed from the fourth car to the last car in the left-turn saturate flow;

$v_{i}$ : the speed of the car when it passes through the pedestrian crossing;

$n$ : number of cars.

367 eligible saturation flow speeds are collected from the 720 left-turn signal cycles at the intersection in the three consecutive workdays. There are 40 saturation flow speeds with no pedestrian impact. The groups of saturation flow speed with inside effects are 139, and outside are 122. The sample size for the saturation speed influenced by pedestrians on both sides is 75 .

\section{Analysis}

It is assumed that all cars in the moving queue followed a trajectory marked for left turning vehicles. Because the demand was greater than or equal to the capacity of the turn lane, the flow was at or near saturation flow status. Thus, the measured speed was the speed at saturation flow rate.

The pedestrian impact on the saturation flow is assessed by the speed and the two variables that are used to describe pedestrian impact, pedestrian relative position to left-turning cars (inside vs. outside and distance to the turning car) and the number of pedestrians at each position. To better assess the impact, the level of pedestrian effect will be defined by the measured average operating speed, based on which saturation flow rate is calculated for each level.

Table 1 lists the speed of left-turn saturation flow with and without pedestrians' interference only on one side (either inside or outside). The average speed of inside effects is $17.9 \mathrm{~km} / \mathrm{h}$ and the average for outside effects is $19.0 \mathrm{~km} / \mathrm{h}$. Pedestrians on the inside of left-turn flow affect the left-turn flow more seriously than those on the outside according to the statisticalanalysis. 
Table 1. Estimated speed with and without pedestrian interference.

\begin{tabular}{cccccc}
\hline Speed & Average & Standard deviation & Min & Max & Median \\
\hline $\mathrm{V}_{\text {flow }}(\mathrm{km} / \mathrm{h})$ & 23.1 & 1.7 & 19.4 & 27.1 & 23.3 \\
Inside $\mathrm{V}_{\text {flow }}(\mathrm{km} / \mathrm{h})$ & 17.9 & 2.3 & 11.5 & 23.7 & 17.4 \\
Outside $\mathrm{V}_{\text {flow }}(\mathrm{km} / \mathrm{h})$ & 19.0 & 2.1 & 15.6 & 24.1 & 19.0 \\
\hline
\end{tabular}

To investigate the effect of numbers of pedestrians, six cases are defined from zero, one, two, three, four and more than four pedestrians.

Figure 3 illustrates the average speed of flow with pedestrians inside and outside the trajectory of turning vehicles by different numbers.

It is clear that the pedestrians' interfering with turning vehicles affect the speed of the left-turn speed at saturation flow seriously but the level of interference does not increase significantly by different number of pedestrians, which is further verified by the Significant Analysis between the adjacent speed groups.

The distance between turning cars and pedestrians is also investigated. The $L_{\text {in }}$ is the distance between the left-turn flow and the pedestrians on the inside (left side) of the flow. Initially, the pedestrian's distance to the turning vehicles is accounted at $0.2 \mathrm{~m}$ intervals, and the speed does not change much between adjacent groups according to the Significant Analysis, and the results of one meter, one point two and etc. meters intervals are the same to the others. However, the speed changes clearly detected when the distance increases to two meters, the difference between the speeds of adjacent groups is significant (Figure 4, Table 2). Thus, there is a significant different effect on the left-turn saturation flow when the distance changes every two meters. Thus, the sensitivity of $L_{\text {in }}$ is "two meters".

The effect on the left-turn saturation flow becomes more seriously with a smaller $L_{i n}$. When the distance is larger than four meters, the effect can be ignored, which have been showed in Figure 4.

The $L_{\text {out }}$ is the distance between the left-turn flow and the pedestrians on the outside (right side). And the sensitivity of $L_{\text {out }}$ is "two meters" also. There are others similar results for the $L_{\text {out }}$ to the results for the $L_{\text {in }}$. The effect on the left-turn saturation flow becomes more serious with a smaller $L$, and the effect can be ignored when the distance is larger than four meters.

The following conclusions can be found so far: the speed of saturation flow is mainly affected by the position of pedestrians (inside or outside of left-turn vehicle's trajectory) and the distance between the vehicle and pedestrians. It can also be concluded that the sensitivity of the distance interval is two meters, which is verified by the Significant Analysis, but the number of pedestrians is not an influential factor.

Thus, the pedestrian influence cases which are studied are shown in Table 3.

Combining all analysis presented previously, the speed at the saturation flow rate estimated by the defined six cases in Table 3. There is no difference between the speed of $S 5$ and the speed of $S 0$. The other four cases show the impact of pedestrian existence. The speed is the lowest with $S 1$, and the highest with $S 4$. As the statistical tests show, the speed difference is significant between $S 4$ and the other three cases $(S 1, S 2$, and $S 3)$. The speed with $S 2$ is significantly different from $S 1$, but the speed with $S 2$ and $S 3$ are not significantly different from the significance test. When $S 2$ and $S 3$ are merged into one group, there is a significant difference between the different groups.

Thus, the speed of left-turn saturation flow, which is affected by the pedestrians on a single side, can be finally classified into four grades: I, II, III, and IV (Table 4).

The speed affected by the pedestrians on both sides is compared with the speed affected by pedestrians on a single side. The following Table 5 defines the analysis case with pedestrians on both sides of the flow simultaneously.

The speed affected by the pedestrians on both sides is compared with the speed affected by pedestrians on a single side. Figure 5 shows that the pedestrians affect the average speed and the effect is the largest when both sides of turning vehicle are presented with pedestrians. Further statistical tests indicate that there is no significant difference between group I and groups $T 11, T 12, T 13$, and the difference in speed between group II and groups $T 21$ and $T 22$ is not significant. The speed difference between group III and group T32 is also not significant, which means that the effect on the left-turn saturation flow by both sides of pedestrians is same to the effect by pedestrians on one serious side. 


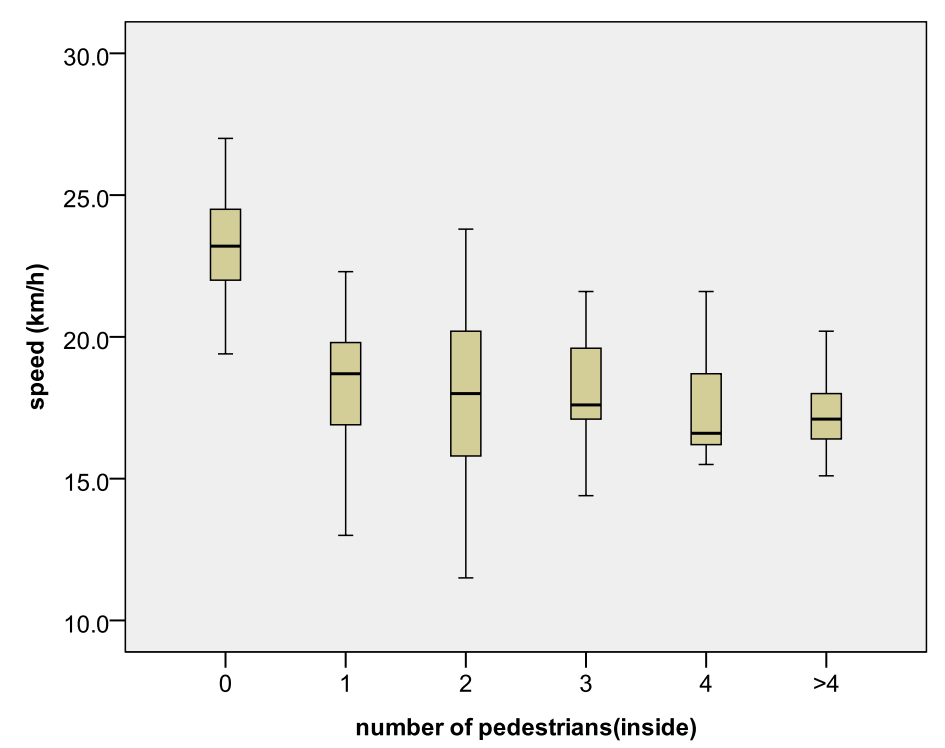

(a)

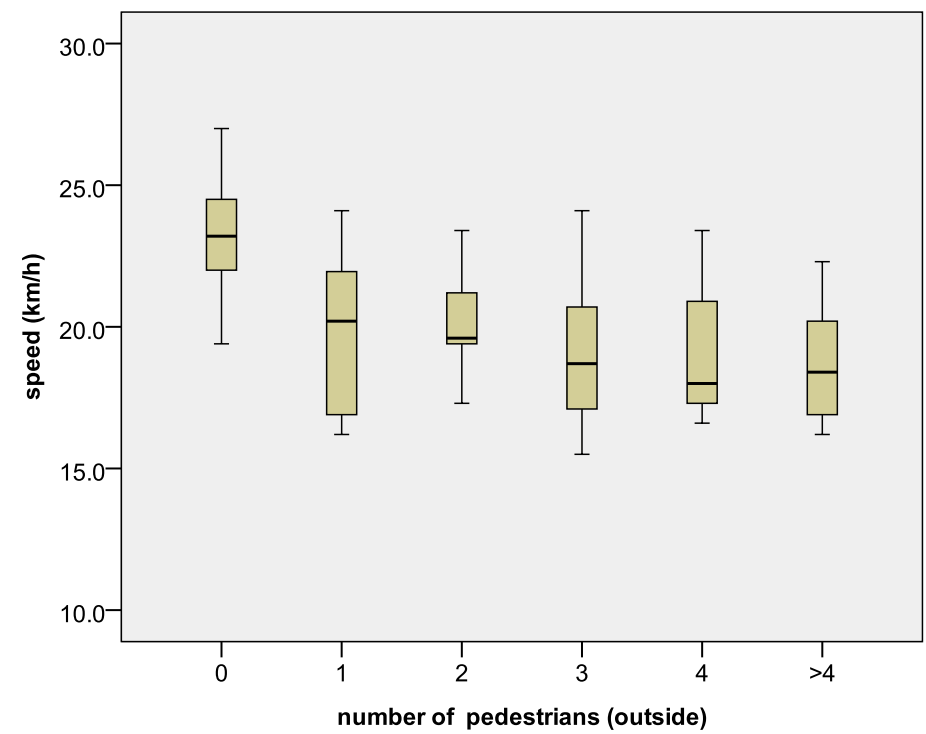

(b)

Figure 3. Speed of flow by pedestrian position and frequency. (a) Inside; (b) Outside.

For practical applications, the pedestrian impact on the left-turn flow is classified into four levels shown in Table 6. Each level can be distinguished quantitatively using the mean difference between groups which is calculated in the statistical significance tests.

In order to verify the effect on the speed of saturation flow by pedestrians, another signal intersection is investigated (intersection B). The first signal intersection described above is intersection A. The speed of saturation flow at intersection B is $20.6 \mathrm{~km} / \mathrm{h}$, which is smaller than intersection A: $23.1 \mathrm{~km} / \mathrm{h}$. With different level of effect, the decreases of the speed of saturation at A and B are different. However, the speed reduction ratio is almost the same, which is showed in Table 7.

Therefore, the speed reduction ratio is suitable for representing the impact to the saturation flow by pedestrians.

Tine headway between vehicles has been surveyed, and the saturation flow rate has been calculated. Table 8 displays the results of influences on the speed and saturation flow rate at intersection A. 


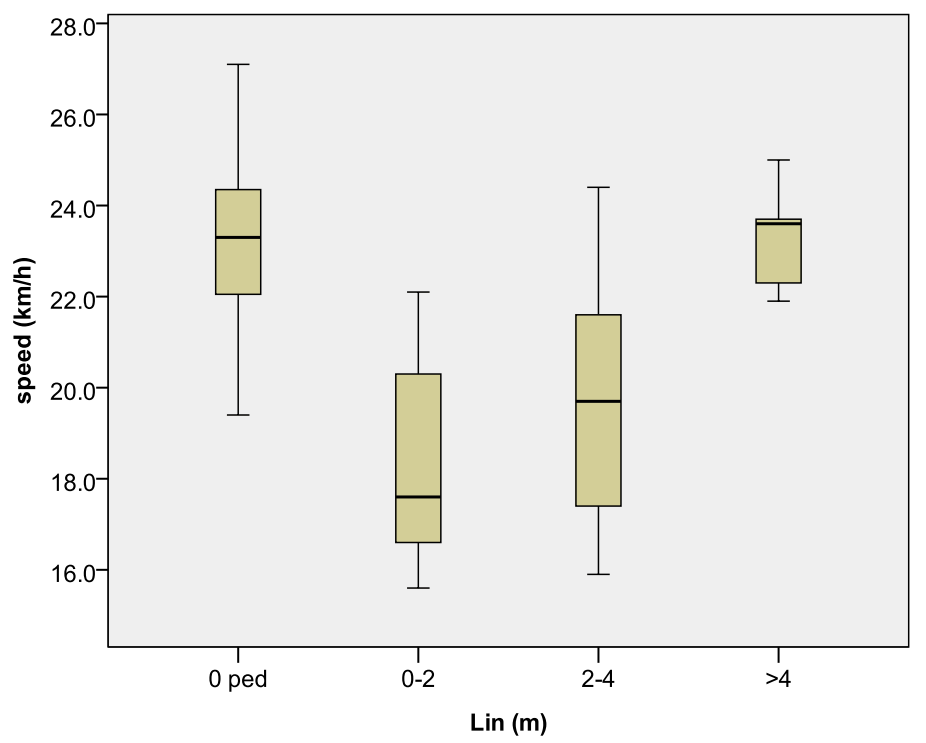

Figure 4. Speed of saturation flow affected by inside pedestrians with different $L_{\text {in }}(\Delta L=2 \mathrm{~m})$.

Table 2. Significant Analysis for the effect on the left-turn saturation flow by pedestrians with different $L_{\text {in }}(\Delta L=2 \mathrm{~m})$.

\begin{tabular}{cccc}
\hline Group & Comparison & Significance level & Significant or not \\
$L_{\text {in }}=0$ & $L_{\text {in }}=0-2$ & 0.000 & Yes \\
& $L_{\text {in }}=2-4$ & 0.000 & Yes \\
$L_{\text {in }}>4$ & 0.727 & No \\
$L_{\text {in }}=0-2$ & $L_{\text {in }}=0$ & 0.000 & Yes \\
& $L_{\text {in }}=2-4$ & 0.007 & Yes \\
$L_{\text {in }}=2-4$ & $L_{\text {in }}>4$ & 0.000 & Yes \\
& $L_{\text {in }}=0$ & 0.000 & Yes \\
& $L_{\text {in }}=0-2$ & 0.007 & Yes \\
& $L_{\text {in }}>4$ & 0.000 & Yes \\
$L_{\text {in }}>4$ & $L_{\text {in }}=0$ & 0.727 & No \\
& $L_{\text {in }}=0-2$ & 0.000 & Yes \\
& $L_{\text {in }}=2-4$ & 0.000 & Yes \\
\hline
\end{tabular}

Table 3. Study cases.

\begin{tabular}{cccc}
\hline Case & Description & Case & Description \\
\hline$S 0$ & No pedestrians & $S 3$ & Outside pedestrians, $L=0-2 \mathrm{~m}$ \\
$S 1$ & Inside pedestrians, $L=0-2 \mathrm{~m}$ & $S 4$ & Outside pedestrians, $L=2-4 \mathrm{~m}$ \\
$S 2$ & Inside pedestrians, $L=2-4 \mathrm{~m}$ & $S 5$ & On both sides, $L>4$ \\
\hline
\end{tabular}


Table 4. Study cases.

\begin{tabular}{cc}
\hline Group & Description \\
\hline S0 & No pedestrians \\
I (S1) & Inside pedestrians, $L_{\text {in }}=0-2 \mathrm{~m}$ \\
II (S2, S3) & Inside pedestrians, $L_{\text {in }}=2-4 \mathrm{~m}$, or outside pedestrians, $L_{\text {out }}=0-2 \mathrm{~m}$; \\
III (S4) & Outside pedestrians, $L_{\text {out }}=2-4 \mathrm{~m}$ \\
IV (S5) & $L>4$; no effect \\
\hline
\end{tabular}

Table 5. Case definition for pedestrians on both sides of turning vehicles.

\begin{tabular}{ccc}
\hline Case Identifier & $L_{\text {in }}$ (left) & $L_{\text {out }}$ (right) \\
\hline$T 11$ & & $0-2(1)$ \\
$T 12$ & $0-2(1)$ & $2-4(2)$ \\
$T 13$ & $>4(3)$ \\
\hline$T 21$ & & $0-2(1)$ \\
$T 22$ & $2-4(2)$ & $2-4(2)$ \\
$T 23$ & & $>4(3)$ \\
$T 31$ & & $0-2(1)$ \\
$T 32$ & $>4(3)$ & $2-4(2)$ \\
$T 33$ & & $>4(3)$ \\
\hline
\end{tabular}

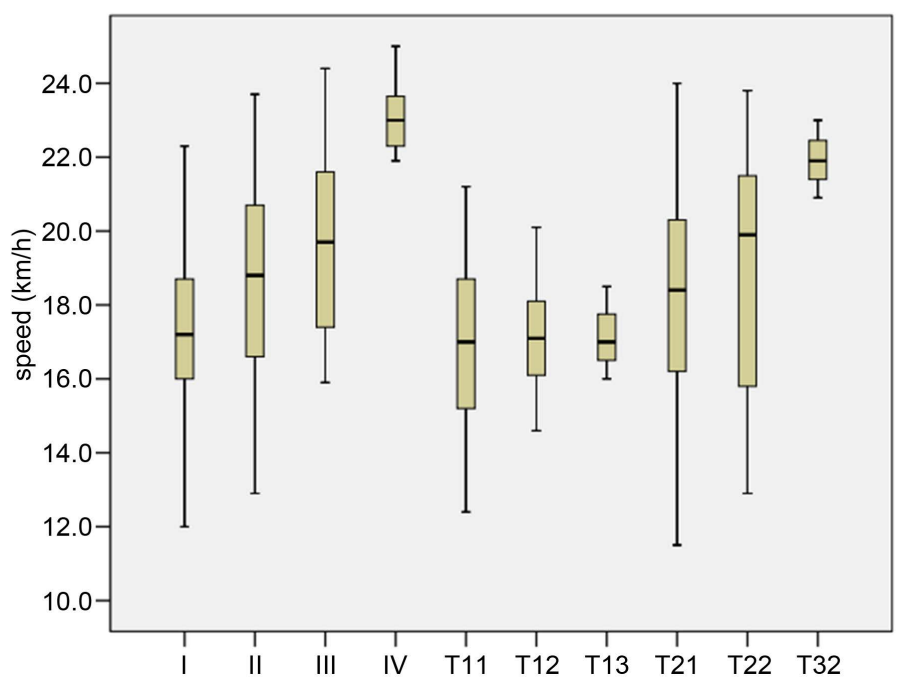

Figure 5. Speed vs. defined cases.

\section{Discussion}

As shown, the situation described by level I (serious impact) is that there are pedestrians on the inside of the leftturn saturation flow and the distance is less than two meters. At this time, the saturation flow rate is decreased by $15.7 \%$.

If there are pedestrians on the inside of the left-turn saturation flow and the distance is between two to four meters, or there are pedestrians on the outside of the left-turn saturation flow and the distance is less than two 
Table 6. Level of pedestrian effect.

\begin{tabular}{cccc}
\hline Impact level & Description & Pedestrians on single side & Estimated saturate speed \\
\hline Level I & Seriously & Inside pedestrians $L_{\text {in }}=0-2 \mathrm{~m}$ & $<18.0 \mathrm{~km} / \mathrm{h}$ \\
Level II & Moderately & Inside pedestrians, $L_{\text {in }}=2-4 \mathrm{~m}$, or outside pedestrians, $L_{\text {out }}=0-2 \mathrm{~m} ;$ & $18.0-19.8 \mathrm{~km} / \mathrm{h}$ \\
Level III & Generally & Outside pedestrians, $L_{\text {out }}=2-4 \mathrm{~m}$ & $19.8-21.6 \mathrm{~km} / \mathrm{h}$ \\
Level IV & Barely & $L>4 \mathrm{~m}$ & $>21.6 \mathrm{~km} / \mathrm{h}$ \\
No pedestrian & No effect & No pedestrian & $23.0 \pm 1.5 \mathrm{~km} / \mathrm{h}$ \\
\hline
\end{tabular}

Table 7. Speed reduction ratio under different pedestrian impact level.

\begin{tabular}{ccc}
\hline Pedestrian impact level & Speed reduction ratio (A) & Speed reduction ratio (B) \\
\hline Level I & $>21.7 \%$ & $>22.3 \%$ \\
Level II & $13.9 \%-21.7 \%$ & $15.0 \%-22.3 \%$ \\
Level III & $6.1 \%-13.9 \%$ & $7.7 \%-15.0 \%$ \\
Level IV & $<6.1 \%$ & $<7.7 \%$ \\
\hline
\end{tabular}

Table 8. Speed and left-turn saturation flow with different level of effect (intersection A).

\begin{tabular}{|c|c|c|c|c|}
\hline Impact level & $\begin{array}{l}\text { Speed of saturation } \\
\text { flow }(\mathrm{km} / \mathrm{h})\end{array}$ & $\begin{array}{l}\text { Headway of saturation } \\
\text { flow (s) }\end{array}$ & Saturation flow (puc/h) & $\begin{array}{l}\text { Reduction to the } \\
\text { saturation flow (\%) }\end{array}$ \\
\hline Level I & $<18.0 \mathrm{~km} / \mathrm{h}$ & $2.36 \pm 0.15$ & 1525 & $15.7 \%$ \\
\hline Level II & $18.0-19.8 \mathrm{~km} / \mathrm{h}$ & $2.23 \pm 0.10$ & 1614 & $10.7 \%$ \\
\hline Level III & $19.8-21.6 \mathrm{~km} / \mathrm{h}$ & $2.11 \pm 0.07$ & 1706 & $5.8 \%$ \\
\hline Level IV & $>21.6 \mathrm{~km} / \mathrm{h}$ & $2.05 \pm 0.05$ & 1756 & $3.0 \%$ \\
\hline No effect & $23.0 \pm 1.5 \mathrm{~km} / \mathrm{h}$ & $1.99 \pm 0.05$ & 1809 & - \\
\hline
\end{tabular}

meters, the impact is moderate and the level is II. Compared with impact level I, this also indicates that pedestrians on the inside affect the left-turn flow more seriously than those on the outside.

When left-turn saturation flow is affected by pedestrians, the minimum headway of the left-turn saturation flow is increased by $3 \%-15.7 \%$ (level IV to level I). This is less than but similar to the statistical result of $14 \%$ $20 \%$ (Wei etc., 2003). The saturation flow rate is decreased by $15.7 \%$ when the left-turn flow is impacted seriously by pedestrians. At that time, the pedestrians are on the inside of the left-turn flow and the distance is less than two meters, and the speed of left-turn saturation flow is less than $18.0 \mathrm{~km} / \mathrm{h}$ (Table 8). The Reduction Factor in the "Code for design of urban road engineering" (CJJ37-2012) is 0.9, this corresponds to the level II (moderately) in this paper.

The results also show that while the impact of pedestrian crossing during a protected left-turn phase on the saturation flow rate is significant, the actual number of pedestrians does not make a difference. Based on the data analysis, higher pedestrian crossing volume could result in a smaller distance between vehicles and pedestrians. In that aspect, pedestrian volume can affect vehicular speed or saturation flow rate indirectly.

\section{Conclusions}

By investigating how the saturation flow rate is affected by illegal pedestrian crossings, this paper analyzes the imagery data collected at two intersections in Beijing, which show that pedestrians on the inside of vehicular flow affect the left-turn saturation rate the most. The distance between pedestrians and the left-turn vehicles is a significant influential factor, while the number of pedestrian is not. If the distance is larger than four meters, the pedestrian impact can be ignored, but if the distance is within four meters, the pedestrian effect is larger when 
the distance is smaller. Based on the statistical analysis, the pedestrian influence is classified into four levels and the effect is quantitatively estimated at each level. The saturation flow rate can be reduced by $15.7 \%$ with pedestrian crossings.

The results show that for highly congested urban intersections, it is critical to enforce traffic control for pedestrians. Widely installed video cameras at urban intersections have effectively reduced irregular vehicle operations but illegal pedestrian's crossing is largely ignored in China. The results of this study demonstrate how important it is to regulate pedestrian flow at signalized intersections.

Effectively stopping illegal pedestrian crossings needs innovative traffic control and enforcement methods since such pedestrian behaviors are commonly observed everywhere. The barrier installed between travel lanes and sidewalks in recent years in Beijing has effectively stopped pedestrians from crossing urban street. Similar ideas can be utilized at intersections, for instance, allowing pedestrians to move to the center of an intersection just a few meters away from left-turning vehicles at the start of the protected left-turn phase.

This study did not take into account the intersections' geometry; it will be a future study topic by the authors.

\section{References}

[1] Viney, N.D. and Pretty, R.L. (1982) Saturation Flow of a Movement Subject to a Pedestrian Stream at Traffic Signals. Proceedings of the Eleventh Australian Road Research Board Conference, University of Melbourne, 23-27 August 1982, 157-166.

[2] Coeymans, J.E. and Herrera, J.C. (2003) Estimating Values for Traffic Parameters in Turning Lanes. Journal of the Transportation Research Board, 1852, 47-54. http://dx.doi.org/10.3141/1852-07

[3] Chen, X.M. (2008) Theoretical Research for Capacity of Signalized Intersection on Urban Road Network in Mixed Traffic Condition .Beijing Jiaotong University, Beijing, 49-55.

[4] Xu, J.Q. and Chen, X.W. (2008) Fundamentals of Traffic Engineer. 3rd Edition, China Communication Press, Beijing, $120-121$.

[5] Wei, H. and Ren, F.T. (1993) Non-Motorized Transport Interference in Motor Vehicle Flow at Urban Intersections. Journal of Beijing University of Technology, 19, 72-79.

[6] Wei, H., Lu, F., Hou, G. and Mogharabi, A. (2003) Nonmotorized Interference and Control Measures at Signalized Intersections in China. Journal of the Transportation Research Board, 1846, 44-49. http://dx.doi.org/10.3141/1846-08

[7] Transportation Research Board (2010) Highway Capacity Manual. National Research Council, Washington DC.

[8] Xu, L.J. and Wang, W. (2006) Analysis of Influence of Left-Turn Non-Motors in Signalized Intersection. China Journal of Highway and Transport, 19, 89-92.

[9] Chen, Z.Q. (2009) Analysis and Modeling on Characteristics of Through Vehicles’ Movement at Signalized Intersections with Mixed Traffic. Beijing Jiaotong University, Beijing, 65-80.

[10] Qian, D.L., Jiang, H.F., Huang, D., et al. (2006) On the Effect of Interference Caused by Mixed Traffic at Signalized Intersection. Journal of Transportation Systems Engineering and Information Technology, 6, 75-78.

[11] Varhelyi, A. (1998) Drivers’ Speed Behavior at a Zebra Crossing: A Case Study. Accident Analysis and Prevention, 30, 731-743. http://dx.doi.org/10.1016/S0001-4575(98)00026-8 
Scientific Research Publishing (SCIRP) is one of the largest Open Access journal publishers. It is currently publishing more than 200 open access, online, peer-reviewed journals covering a wide range of academic disciplines. SCIRP serves the worldwide academic communities and contributes to the progress and application of science with its publication.

Other selected journals from SCIRP are listed as below. Submit your manuscript to us via either submit@scirp.org or Online Submission Portal.
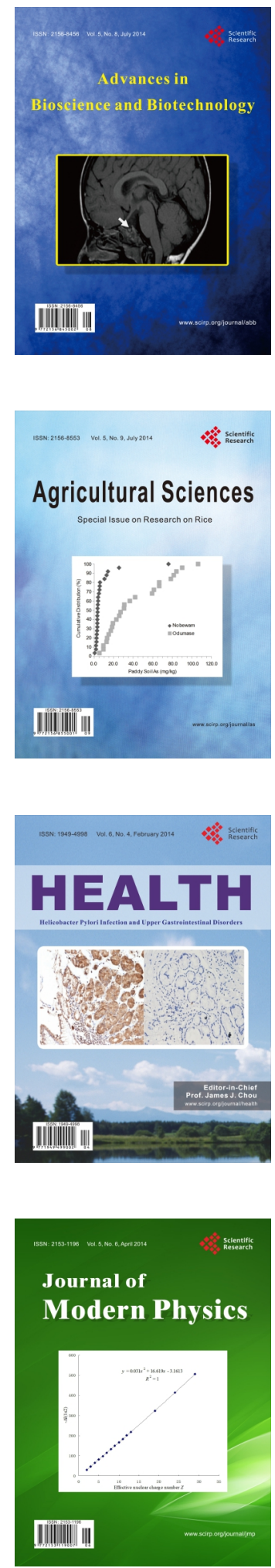
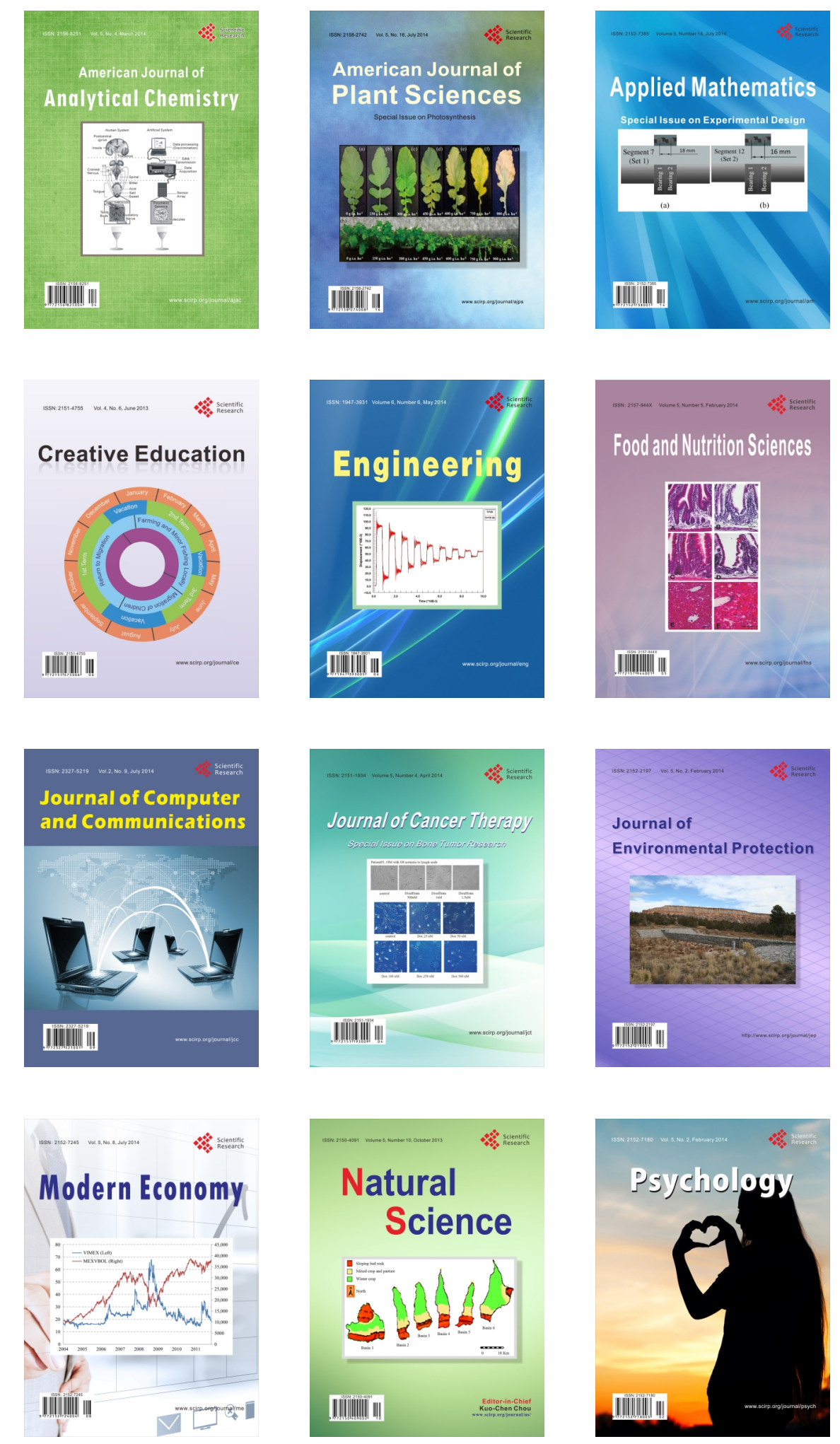